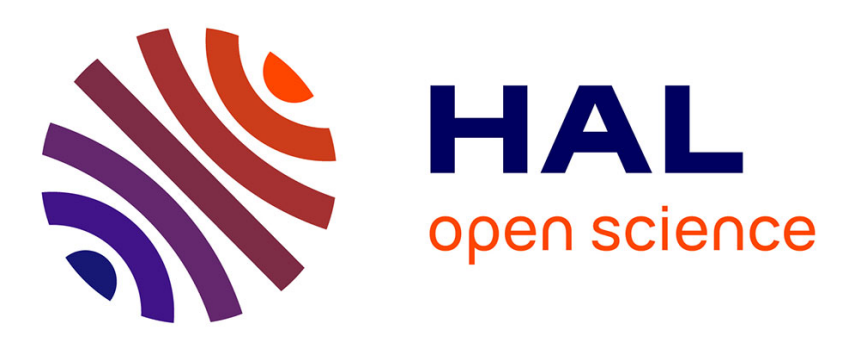

\title{
Selective attention effects on early integration of social signals: Same timing, modulated neural sources
}

\author{
Marwa El Zein, Lucile Gamond, Laurence Conty, Julie Grèzes
}

\section{To cite this version:}

Marwa El Zein, Lucile Gamond, Laurence Conty, Julie Grèzes. Selective attention effects on early integration of social signals: Same timing, modulated neural sources. NeuroImage, 2014, 10.1016/j.neuroimage.2014.10.063 . hal-01079736

\section{HAL Id: hal-01079736 \\ https://hal.sorbonne-universite.fr/hal-01079736}

Submitted on 3 Nov 2014

HAL is a multi-disciplinary open access archive for the deposit and dissemination of scientific research documents, whether they are published or not. The documents may come from teaching and research institutions in France or abroad, or from public or private research centers.
L'archive ouverte pluridisciplinaire HAL, est destinée au dépôt et à la diffusion de documents scientifiques de niveau recherche, publiés ou non, émanant des établissements d'enseignement et de recherche français ou étrangers, des laboratoires publics ou privés. 


\section{Selective attention effects on early integration of social signals:}

\section{same timing, modulated neural sources.}

Marwa El Zein ${ }^{1,2}$; Lucile Gamond ${ }^{1,3}$; Laurence Conty ${ }^{1,4}$ and Julie Grèzes ${ }^{1,5 \#}$

${ }^{1}$ Laboratoire des Neurosciences Cognitives (LNC), INSERM U960, Institut d'Etudes Cognitives, Ecole Normale Supérieure, Paris, France.

${ }^{2}$ UPMC Université Paris 06, Paris, France.

${ }^{3}$ UFR de psychologie, Université Paris 8, Saint-Denis 93526 cedex, Paris, France.

${ }^{4}$ Laboratoire de Psychopathologie and Neuropsychologie (LPN, EA2027), Université Paris 8, Saint-Denis, France.

${ }^{5}$ Centre de Neurolmagerie de Recherche, CRICM, Université Pierre et Marie Curie-Paris 6 UMR-S975, Inserm U975, CNRS UMR7225, Groupe Hospitalier Pitié-Salpêtrière, Paris, France.

Running title: Selective attention effects on the integration of social signals.

\section{"Correspondence:}

Marwa El Zein \& Julie Grèzes

Cognitive Neuroscience Lab., INSERM U960

IEC, Ecole Normale Supérieure,

29 Rue d'Ulm, 75005 Paris, France.

Tel: +331443226 76. Fax: +33144322686.

Email: marwa.el.zein@ens.fr, Julie.grezes@ens.fr 


\section{ABSTRACT}

Humans combine co-emitted social signals to predict other's immediate intentions and prepare an adapted response. However, little is known about whether attending to only one of co-emitted social signals impacts on its combination with other signals. Here, using electroencephalography, we address selective attention effects on early combination of social signals. We manipulated three visual cues: gaze direction, emotional expression, and pointing gesture, while participants performed either emotion or gaze direction judgments. Results showed that a temporal marker of social cues integration emerges $170 \mathrm{~ms}$ after the stimulus onset, even if the integration of the three visual cues was not required to perform the task, as only one feature at a time was task-relevant. Yet, in addition to common temporal regions, the relative contribution of specific neural sources of this integration changed as a function of the attended feature: integration during emotion judgments was mainly implemented in classical limbic areas but in the dorsal pathway during gaze direction judgments. Together, these findings demonstrate that co-emitted social cues are integrated as long as they are relevant to the observer, even when they are irrelevant to the ongoing task.

Keywords: Attentional influence, EEG, integration, self-relevance, social cues. 


\section{INTRODUCTION}

The quality of our social interactions depends on correctly detecting co-emitted social signals and adapting our behaviour accordingly. Of interest, once merged into a single percept, co-emitted social signals can take on new significance and certain combinations, notably those indicating the presence of threat, become more relevant than others. Typically, angry expressions are perceived as more threatening when associated with a direct gaze than with an averted gaze (Sander et al. 2007; N'Diaye et al. 2009; Sato et al. 2010; Conty et al. 2012). Despite the clear relevance of understanding the spatiotemporal characteristics of the mechanisms underlying the combination of social cues, the question of whether, when and how, directing one's attention towards a specific social signal impacts on its neural integration with other co-emitted social cues remains.

Indeed, the literature exploring the neural sources of gaze and expression integration reports incongruous results (Graham and Labar 2012). One possible explanation is that the neural sources underlying social cues integration may be task demand-dependent (Graham and Labar 2012; Dumas et al. 2013). While most of the functional Magnetic Resonance Imagery (fMRI) experiments revealed that the amygdala integrated emotion and gaze when participants were required to attend to the emotional content of the faces or their gender (Sato et al. 2004, 2010; Sander et al. 2007; N'Diaye et al. 2009; Adams et al. 2012), the premotor cortex was involved in gaze and expression combination when participants were requested to attend to gaze direction (Conty et al. 2012). Electroencephalography $(\mathrm{EEG}$ ) results also suggest that the temporal marker of such integration is susceptible to task demand influences: it emerges around $170 \mathrm{~ms}$ after stimulus onset when participants attend emotional expressions (Akechi et al. 2010), at around 200 ms when participants attend gaze direction (Conty et al. 2012), at 240 ms during passive viewing (Rigato et al. 2010), and later during an oddball task concerning gaze direction (Klucharev and Sams 2004). Finally, other studies in the literature have reported that when observers orient their attention to only one feature at a time as requested by the task (i.e. judging the emotion or the gaze of emitters), co-emitted facial 
signals are processed independently (Bindemann et al. 2008) by anatomically and functionally segregated neural structures, at least initially (Klucharev and Sams. 2004; Pourtois et al. 2004).

To directly address how selective attention affects the time course and neural bases of early combination of social signals, we implemented an electroencephalography (EEG) experiment (evoked potentials and source reconstruction analysis). To parametrically modulate the self-relevance of perceived social signals, we manipulated three visual cues: emotion, gaze direction and pointing gesture (as in Conty et al. 2012). Participants performed two tasks: one which requested them to attend only to the gaze direction cue and one which requested them to attend only to the emotional cue. Based on our previous study using the same stimuli (Conty et al. 2012), we expected an early combination (rather than parallel processing) of the social cues that clearly portrayed a relevant threat for the observer within the first $200 \mathrm{~ms}$. Yet, how the precise temporality and the sources of this integration were influenced by task-demand was addressed in a hypotheses-free manner.

\section{MetHODS}

\section{Participants}

Eighteen healthy subjects (10 females; mean age, $24.0 \pm 0.5$ years) participated in the EEG experiment. All participants were right handed, with a normal or corrected vision, and were free of current or past psychiatric or neurological disorders. The subjects gave their written informed consent and were paid for their participation. The study was approved by the local Ethics Committee and was conducted in accordance with the Declaration of Helsinki.

\section{Stimuli}

The stimuli used in the present experiment were developed by Conty and Grèzes (2011). They consisted of 192 photographs of 12 actors (six females) seen under 8 conditions: 2 emotions (anger / neutral) * 2 gaze directions (head and eye gaze directed toward the participant or averted to the 
right and left side: direct/averted) * 2 gestures (pointing / no pointing) (Fig. 1a). An "initial position" photograph depicts each of the actors with a neutral expression, arms by their sides, and an intermediate head and eye direction of $15^{\circ}$. More detailed description of the stimuli can be found in Conty and Grèzes. (2011).

\section{Procedure}

Each trial started with a fixation screen $(500 \mathrm{~ms})$ consisting of a central red fixation point and four red angles, displayed on a uniform gray background. The participant was instructed to fixate the central point and to keep his/her attention inside the fixation area at the level of the central point throughout the trial, avoiding eye blinks and saccades. Because dynamic social displays are more ecologically valid (Sato et al., 2010; Schilbach, 2010), we created an apparent movement by presenting two photographs, one after the other (Conty et al. 2007, 2012). The first photograph always displayed the actor in the initial position during a random period of time, ranging from 1200 to $1500 \mathrm{~ms}$. The second one immediately followed and displayed the same actor in one of the eight conditions of interest (Fig. 1b) for 1300 ms. Throughout the trial, the actor's face remained within the fixation area. Then, a response screen was presented for $1000 \mathrm{~ms}$, followed by a black screen of 500 ms preceding the next trial. We delayed the responses to ensure that the participants responded at the end of the observation period.

The experiment was divided in 8 experimental blocks of 96 trials each. Two tasks alternated in separate blocks resulting in 4 blocks for each task. Participants were requested to focus either on the nature of the emotional expression (expression task) or the gaze direction of the actor (direction task). We chose to only include those two tasks based on the literature described in the introduction.

The expression task consisted in indicating the content of the actors' facial expressions during a response screen displaying the words "neutral" and "angry". In the direction task, participants had to indicate the gaze direction during a response screen now displaying the words "me" and "other". The location of the two words (on the right or left parts of the screen) was randomized (Fig. 1b). Varying 
the location of the words allowed preventing the participants from preparing their responses during the observation period. Therefore, our design was not appropriate for detecting any reaction time advantage in making judgments about gaze directions or emotions. During the EEG experiment, the participants perfectly performed the two tasks (Expression task: mean correct response $=96 \pm 0.8 \%$ and Direction task: mean correct response $=98 \pm 0.4 \%$ ), which assured that participants kept their attention on the stimuli.

To summarize, there were 16 conditions of interest in our experiment: 2 Tasks (Expression/Direction) * 2 Emotions (Anger/Neutral) * 2 Gaze directions (Direct/Averted) * 2 Gestures (Pointing/No pointing).

\section{Post-test}

On the same day, just after the EEG experiment, participants had to perform a behavioural post-test. They were exposed to all stimuli using the same procedure described above. After each trial, they had to evaluate their felt self-involvement on a scale ranging from 0 to $9(0$, "not involved"; 9 , "highly involved"). A repeated-measures ANOVA was performed on these subjective ratings, with emotion (anger/neutral), gaze direction (direct/averted) and gesture (pointing/no pointing) as within-subjects factors.

\section{EEG data acquisition, processing and analyses}

The recording of the EEG activity was held at Centre de Neurolmagerie de Recherche (CENIR at Institut du Cerveau et de la Moelle Epinière; ICM) at a sampling rate of $1000 \mathrm{~Hz}$ using a BRAINAMP amplifier (Brain Products, BRAINAMP MR PLUS). Participants were equipped with an EEG cap of 63 sintered $\mathrm{Ag} / \mathrm{AgCl}$ ring electrodes (Easycap). The reference channel was placed on their nose and a forehead ground was used. Impedances were kept under a threshold of $10 \mathrm{k} \Omega$. EEG signal was low pass filtered online at $250 \mathrm{~Hz}$.

The raw EEG data was recalculated to average reference, down-sampled to $500 \mathrm{~Hz}$, and low-pass filtered at $30 \mathrm{~Hz}$ (using EEGlab version 11 sccn.ucsd.edu/eeglab). Trials containing blinks and muscles 
artefacts were manually excluded. We computed the ERPs for each condition between $200 \mathrm{~ms}$ before and $800 \mathrm{~ms}$ after the onset of the second photograph and corrected using a baseline subtraction (from $-200 \mathrm{~ms}$ to stimulus onset).

Four classical ERP components were studied: the occipital P100, the temporal N170, the central P200 (Ashley et al. 2004; Vlamings et al. 2009) and a later component: the late positive potential (LPP) (Cuthbert et al. 2000; Schupp et al. 2000). For each ERPs of interest, we selected specific electrodes and time range on the peak amplitude observed on mean ERPs computed on all trials. For the P100, average activity on two occipito-parietal electrodes around the peak, between 92 and $116 \mathrm{~ms}$, in each hemisphere was selected (01/PO7, O2/PO8). For the N170, average activity on three electrodes around the peak, between 162 and 186 ms, in each hemisphere was selected (P7/TP7/TP9, P8/ TP8/TP10). For the P200, average activity on six central electrodes around the peak, between 184 and $208 \mathrm{~ms}$, was selected (C1/Cz/C2/CP1/CPZ/CP2). Finally, according to previous literature on the late positive potential (LPP; Schupp et al. 2000; Liu et al. 2012), LPP related activity was measured by considering the mean activity averaged on five parietal electrodes (on which the LPP activity was maximal) between 300 and $700 \mathrm{~ms}(\mathrm{P} 3, \mathrm{P} 1, \mathrm{Pz}, \mathrm{P} 2$ et $\mathrm{P} 4)$.

Repeated-measures ANOVA was performed on each ERP measure with task (expression/direction), emotion (anger/neutral), gaze direction (direct/averted), gesture (no pointing/pointing), - and for bilateral ERP components: P100, N170 - hemisphere (right/left), as within-subjects factors.

\section{EEG source localization}

Brainstorm was used for source reconstruction (Tadel et al. 2011 http://neuroimage.usc.edu/brainstorm). A source model that consisted in 15028 current dipoles was used to obtain a cortical current source density mapping per subject and condition. Dipole orientations were loosely constrained to the cortical mantle of a generic brain model taken from the standard Montreal Neurological Institute (MNI) template brain provided in Brainstorm. This head model was warped to the standard geometry of the EEG sensor cap taking into account a mean 
polhemus of 4 subjects. Computation of the forward model of EEG was done using the OpenMEEG BEM model (Kybic et al. 2005; Gramfort et al. 2010). For each subject and each condition, cortical current maps were computed from the ERPs of interest using the weighted minimum norm current estimate (WMNE).

First, to estimate the source of the integration of emotion, gaze and gesture observed on the N170, irrespective of the tasks, we averaged the sources data on the time window of the N170 (162-186 ms) for each subject and condition, and across tasks. Then, we applied a statistical mask on every vertex of the 15028 vertices of the model that reflects our parametric increase of self-relevance as a function of the number of self-oriented social cues. A vertex was thus considered as significant if: 1 ) the activity of the most self-relevant condition (Anger, Direct and Pointing) was statistically different (t-tests) from the activity in all the other conditions; and if 2) the activity in the two conditions with two self-directed cues (Anger, Direct, no Pointing and Neutral, Direct, Pointing) were statically different (t-tests) from the activity in the condition with one self-directed cue (Neutral, Direct, no Pointing).

Secondly, to address selective attention effects on the sources of early social cues integration, we performed the same masked analysis as described above, but for each task separately. We created a conjunction map to identify neural sources that were common to both tasks, and then used this conjunction image as an exclusive mask to identify the sources that were specific to one or the other task.

For both analyses, regions representing a surface area superior to $1.5 \mathrm{~cm}^{2}$ (and thus included more than 15 contiguous vertices), following the self-relevance pattern are reported and labelled on the basis of the correspondence between the Tzourio-Mazoyer brain atlas (Tzourio-Mazoyer et al. 2002), the brainstorm generic brain and source model implemented in Brainstorm software. For each region, we reported the number of vertices observed (Table 1). We selected 3 regions: 1) one revealed across tasks, 2) one for the Direction task and 3) one for the Expression task, and plotted for 
comparison the data for one representative vertex of those regions, for each task independently (Fig.

3).

\section{RESULTS}

\section{Behavioural post-test}

As expected from our previous experiment (Conty et al., 2012), stimuli were considered more selfinvolving in the angry condition as compared with the neutral condition $\left(F_{(1,17)}=32.8, p<0.001\right)$, in the direct gaze condition as compared with the averted gaze condition $\left(F_{(1,17)}=160.9, p<0.001\right)$, and in the pointing condition as compared with the no pointing condition $\left(F_{(1,17)}=69.7, p<0.001\right)$. Interestingly, an interaction between emotion, gaze, and gesture was observed $\left(F_{(1,17)}=10.3, p<0.01\right)$. Post-hoc comparisons revealed that the condition that was judged as more self-involving as compared to the other conditions (all $t_{(1,17)}>3$, all $p<0.001$ ) was the condition where the 3 relevant cues were coemitted (angry expression, direct gaze and pointing gesture). Moreover, the self-involvement ratings increased with the number of self-directed social cues: it was higher when two self-directed social cues were present (direct gaze and anger or direct gaze and pointing) as compared to one selfdirected cue (direct gaze) (all $t_{(1,17)}>5.7$, all $p<0.001$ ) whereas no difference was observed between the two conditions of two self-directed social cues $\left(t_{(1,17)}=0.09, p=0.92\right)$ (Fig. 2a).

\section{Time course of social cues processing}

We addressed when emotion, gaze, and gesture were integrated and whether this integration varied with task demand. On P100 activity, we observed main effects of emotion and gesture. Anger $\left(F_{(1,17)}=17.7, p<0.001\right)$ and pointing $\left(F_{(1,17)}=17,3, p<0.001\right)$ induced greater positive activity than neutral and no-pointing conditions, respectively (see Figure S1). No other main effects or interaction between factors were observed (all $F<1$, all $p>0.2$ ). 
On the temporal N170, main effects of hemisphere, emotion, gaze and gesture were detected. The activity was more important in the right than in the left hemisphere $\left(F_{(1,17)}=6.69, p<0.05\right)$. Anger $\left(F_{(1,17)}=128.04, p<0.001\right)$, direct gaze $\left(F_{(1,17)}=6.17, p<0.05\right)$, and pointing $\left(F_{(1,17)}=24.99, p<0.001\right)$ conditions generated greater negative peak amplitudes than, respectively, neutral, averted gaze, and no-pointing conditions. There was an interaction between emotion and gaze $\left(F_{(1,17)}=6.69, p<0.05\right)$, an interaction between emotion and gesture $\left(F_{(1,17)}=16.3 p<0.001\right)$, and more importantly, an interaction between emotion, gaze, and gesture $\left(F_{(1,17)}=4.59, p<0.05\right)$. This interaction was explained by an interaction between gaze and gesture only in the anger conditions $\left(F_{(1,17)}=4.537, p=0.048\right)$ but not in the neutral conditions $\left(F_{(1,17)}<0.001, p=0.991\right)$, strongly supporting the idea that there was integration between the three co-emitted cues rather than additive effects only. The peak amplitude of the most self-relevant condition (anger, direct gaze and pointing) was greater than all other conditions (all $t_{(1,17)}>3.3$, all $p<0.01$ ) (Fig. 2c). In addition, just as in the post-test ratings, N170 activity increased with the number of self-directed social cues: activity was greater when two self-directed social cues were present (direct gaze and anger or direct gaze and pointing) as compared to one self-directed cue (direct gaze) $\left(t_{(1,17)}=6, p<0.001\right)$ whereas no difference was observed between the two conditions of two self-directed social cues $\left(t_{(1,17)}=0.47, p=0.65\right)$ (Fig. 2 b) .

Analysis ran on the central P200 revealed a main effect of emotion and gaze. Anger $\left(F_{(1,17)}=8.9\right.$, $p<0.01)$ and averted gaze $\left(F_{(1,17)}=5.1, p<0.05\right)$ elicited greater positive activity than, respectively, neutral and direct gaze conditions (see Figure S1). No other main effects or interactions between factors were observed (all $F<1$, all $p>0.2$ ). Although Conty et al. (2012) found a triple interaction on the P200, our results are not surprising as the triple interaction in this study is already observed on the N170. Greater activity for averted gaze as compared to direct gaze on the P200 replicates the findings of Rigato et al. (2010).

On the late positive potential (LPP), we observed main effects of task and emotion. Anger $\left(F_{(1,17)}=24\right.$, $p<0.001)$ induced greater activity as compared to neutral condition. Expression task $\left(F_{(1,17)}=19.4\right.$, $p<0.001$ ) induced greater activity as compared to Direction task (see Figure S1). An interaction 
between gaze and task was also observed $\left(F_{(1,17)}=4.7, p<0.05\right)$ and was justified by a main effect of gaze only in the Direction task $\left(F_{(1,17)}=6, p<0.05\right)$. In this task, direct gaze induced greater activity as compared to averted gaze whereas no such difference was observed in the Expression task (see Figure S1). These results suggest that, at these later temporal stages, the emotional content of the faces may be always processed in both tasks whereas their gaze direction may only be processed in the Direction task. The present main effect of emotion on the late positive potential (LPP) is consistent with previous results that involve the LPP in explicit emotional processing (Cuthbert et al. 2000) and task switching (Rushworth et al. 2002; Astle et al. 2008).

Crucially, there was no main effect of task before the LPP (all $F<1.52$, all $p>0.23$ ) nor interaction of the task with the other factors (all $F<4.2$, all $p>0.05$ ), suggesting at first view that the integration between gaze, emotion and gesture during the first 200 ms after stimulus onset are independent of selective attention related to the task.

Finally, we tested whether there were between-subjects correlations between the peak amplitudes of the N170 and the behavioral self-involvement ratings (for all the different conditions separately as well as for the differences of interest between conditions). No significant effects emerged (all $r<0.4$, all $p>0.1)$.

\section{Sources reconstruction}

We then explored the brain sources of early integration of emotion, gaze and gesture observed on the N170 (see Fig. 2c) and whether the sources of this integration were influenced by task-demand.

Firstly, we estimated these sources of early social cues integration across tasks. The masked source reconstruction analysis revealed that both structures of the limbic system (such as the amygdala, the hippocampus, the cingulate cortex as well as the ventromedial prefrontal cortex), previously reported in experiments using explicit emotional tasks (Sato et al. 2004, 2010; Sander et al. 2007; N'Diaye et al. 2009; Adams et al. 2012), and the dorsal pathway including the parietal and premotor 
cortex, reported by Conty et al. (2012) that used a gaze direction task, are involved in the early integration of emotion, gaze and gesture and showed an increase of activity with the increase of selfrelevant social cues (same pattern as observed on the N170).

Secondly, as the literature pointed toward inconsistent neural sources of a) gaze and emotion combination, and b) of the N170 (Itier and Taylor. 2004; Eimer 2011), and even though there was no statistical interaction on the N170 activity (evoked potential analysis) between the three manipulated social cues (emotion, gaze and gesture) and the task factor, we tested whether the sources of the N170 varied as a function of the ongoing task. To do so, we performed the abovementioned masked analysis for each task separately. When participants attended to the emotional expressions, the amygdala, the hippocampus and the subgenual cingulate area were involved in the integration of social cues (see Table 1 for a full description of the results) whereas the post-central gyrus and the inferior and superior parietal cortex were detected when participants attended to gaze direction. In addition to these task-specific sources, the fusiform gyrus, middle occipital gyrus, inferior occipital gyrus and middle temporal gyrus which most probably involved the superior temporal sulcus (STS) were involved in both tasks (as revealed by the conjunction analysis).

\section{DISCUSSION}

The aim of the present study was to address whether, when and how, directing one's own attention towards a specific social signal emitted by another person impacts on its neural integration with other co-emitted social signals. Our critical finding was that the early integration of social visual cues is indexed by a temporal marker (N170) that is independent of the attended feature, yet being dependent on the relevance of the combination. While the underlying neural sources of the N170 included both limbic and dorsal pathway structures, their relative contribution was modulated as a function of the attended feature: integration during emotion judgments was mainly implemented in classical limbic areas but in the dorsal pathway during gaze direction judgments. 
The amplitude of the N170 and associated neural sources, as well as of the self-involvement ratings, are maximum when the combination of the co-emitted social cues clearly portrayed a threat for the observer, i.e. when the actor expressed anger, looked, and pointed toward the participant, in agreement with previous findings (Klucharev and Sams, 2004; Akechi et al., 2010; Rigato et al., 2010; Conty et al., 2012). The statistical interactions between, in one hand, the three cues, and on the other hand, gaze and gesture within anger conditions only, suggest that the three co-emitted cues are merged into a single percept rather than triggering additive effects only. These results substantiate that our sensory system is not globally adapted to the statistics of all natural stimuli, but rather evolution has tailored our brain to be specifically optimized to biologically relevant stimuli (Machens et al. 2005).

The temporal marker of the integration of emotion, gaze and gesture emerges $170 \mathrm{~ms}$ after stimulus onset. Importantly, this temporal marker emerges even if the integration of the three visual cues was not required to perform the task, as only one feature at a time was task-relevant. To our knowledge, this is the first study that directly compares the impact of two tasks on the spatiotemporal course of early integration of social cues. This allowed to reveal that early integration appears to be temporally task-related attention independent and thus contradicts the view that orienting attention to only one feature at a time results in processing co-emitted facial signals independently (Pourtois et al. 2004, Klucharev and Sams, 2004; Bindemann et al., 2008). A general attention effect (rather than a selective attention effect) may explain whether this marker emerges within the first $200 \mathrm{~ms}$ or later: indeed, when participants are requested to attend to the stimuli by judging either the emotional expressions (present study, Akechi et al., 2010) or the gaze direction (Conty et al., 2012), the temporal marker of social cues integration emerges earlier (between 170 and $200 \mathrm{~ms}$ ) than when participants observe passively (Rigato et al. 2010) or perform an oddball task (starting at 190 and lasting till 350 ms (Dumas et al., 2013)- but see exception by Klucharev and Sams (2004).

The integration of social visual cues recruited the posterior occipital cortex, the fusiform gyrus (FG) and the superior temporal sulcus (STS) independently of task-demand. In functional imaging studies, 
those areas have been frequently associated with face perception (Kanwisher et al. 1997; Puce et al. 1998; George et al. 1999), notably, the FG preferentially processes the invariant aspects of the face while the STS processes changeable aspects such as the gaze and the mouth (Haxby et al. 2000; Hoffman and Haxby 2000). Moreover, EEG source analyses have either reported the STS (Batty and Taylor. 2003; Henson et al. 2003; Itier et al. 2006; Itier and Taylor, 2004; Watanabe et al. 2003) or the FG (Itier and Taylor. 2002; Rossion et al. 2003; Watanabe et al. 2003) as the main source of the N170. Here, we further showed that these classical neural sources of the N170 (Bötzel et al. 1995; Rossion et al. 2003; Conty et al. 2007) are involved in the early integration of facial expression with coemitted gaze direction and gesture, independently of the attended social cue.

Several limbic areas as well as dorsal pathway structures were also found to be engaged during the early integration of relevant social cues. Yet, in contrast to the above-mentioned areas, the contribution of these specific neural sources appears to be modulated as a function of the attended feature: predominantly sustained by classical limbic areas during emotion judgments, the integration of social cues involved the dorsal pathway during gaze direction judgments. Although most of the brain regions were revealed in previous fMRI studies on gaze-emotion integration (Hadjikhani et al. 2008; N'Diaye et al. 2009; Sato et al. 2010), we further demonstrated, using two tasks, that when participants were requested to attend to the emotional content of the faces, the early integration of emotion, gaze and gesture took mainly place in the temporal pole including amygdala-hippocampal region and the subgenual orbital cortex, brain areas known to be involved in emotional processing (Phillips et al. 2003; Höistad and Barbas, 2008; Fusar-Poli et al. 2009), notably explicit processing (Gur et al. 2002; Keightley et al. 2003; Habel et al. 2007). Nonetheless, these regions were less evidenced in the direction task mainly associated with the postcentral and the parietal cortex involved in gaze perception (Puce et al. 1998; Hoffman and Haxby, 2000). Of interest, using fMRI, Zaki et al. (2012) similarly showed the involvement of specific neural networks as a function of the perceiver's goals, i.e. to attend to the gaze direction or to the emotional state of the actor. Here, we further revealed that the contribution of the limbic system to the integration of social cues was enhanced when 
participants explicitly paid attention to emotional cues but decreased when they paid attention to gaze direction while the contribution of the dorsal pathway increased.

The present study thus reveals that the integration of social cues and not simply the perception of one or the other cues recruits a large brain network around $170 \mathrm{~ms}$ after stimulus onset, which activity is partly modulated by task-demand. Still, the question remains as to whether and how biologically relevant information flows within the whole network at a more fine-grained time scale. From macaque monkeys data, one could speculate that the amygdala may initiate the integration and enhance sensory processing of biologically relevant signals thanks to direct projections to the ventral visual pathway including the FG and the STS (Hadj-Bouziane et al. 2012) and premotor cortex in the dorsal pathway (Avendano et al. 1983). To conclude, by addressing selective attention effects on the spatiotemporal course of social cues integration, we were able to show an influence of taskdemand on the "where" component rather than on the "when" component. Moreover, our results demonstrate that all social cues are encoded even when they are irrelevant to the task, as long as they are relevant to the observer.

\section{ACKNOWLEDGEMENTS}

We wish to warmly thank Florence Bouchet and Laurent Hugueville for their useful technical help. The research was supported by ANR-11-EMCO-00902, ANR-11-0001-02 PSL*, ANR-10-LABX-0087 and by INSERM.

\section{REFERENCES}

Adams RB Jr, Franklin RG Jr, Kveraga K, Ambady N, Kleck RE, Whalen PJ, Hadjikhani N, Nelson AJ. 2012. Amygdala responses to averted vs direct gaze fear vary as a function of presentation speed. Soc Cogn Affect Neurosci 7:568-577. 
Akechi H, Senju A, Kikuchi Y, Tojo Y, Osanai H, Hasegawa T. 2010. The effect of gaze direction on the processing of facial expressions in children with autism spectrum disorder: an ERP study. Neuropsychologia 48:2841-2851.

Ashley V, Vuilleumier P, Swick D. 2004. Time course and specificity of event-related potentials to emotional expressions. Neuroreport 15:211-216.

Astle DE, Jackson GM, Swainson R. 2008. Fractionating the cognitive control required to bring about a change in task: a dense-sensor event-related potential study. J Cogn Neurosci 20:255-267.

Avendano C, Price JL, Amaral DG. 1983. Evidence for an amygdaloid projection to premotor cortex but not to motor cortex in the monkey. Brain Res 264:111-117.

Batty M, Taylor MJ. 2003. Early processing of the six basic facial emotional expressions. Brain Res Cogn Brain Res. 17:613-620.

Bindemann M, Mike Burton A, Langton SRH. 2008. How do eye gaze and facial expression interact? Vis Cogn 16:708-733.

Bötzel K, Schulze S, Stodieck SR. 1995. Scalp topography and analysis of intracranial sources of faceevoked potentials. Exp Brain Res 104:135-143.

Conty L, Dezecache G, Hugueville L, Grèzes J. 2012. Early binding of gaze, gesture, and emotion: neural time course and correlates. J Neurosci 32:4531-4539.

Conty L, Grèzes J, 2011. Look at me, I'll remember you: the perception of direct gaze enhances memory and right hippocampal activity. Human Brain Mapping 33:2428-2440.

Conty L, N'Diaye K, Tijus C, George N. 2007. When eye creates the contact! ERP evidence for early dissociation between direct and averted gaze motion processing. Neuropsychologia 45:3024-3037. 
Cuthbert BN, Schupp HT, Bradley MM, Birbaumer N, Lang PJ. 2000. Brain potentials in affective picture processing: covariation with autonomic arousal and affective report. Biol Psychol 52:95-111.

Dumas T, Dubal S, Attal Y, Chupin M, Jouvent R, Morel S, George N. 2013. MEG Evidence for Dynamic Amygdala Modulations by Gaze and Facial Emotions. PloS One 8:e74145.

Eimer M. 2011. The Face-Sensitive N170 Component of the Event-Related Brain Potential. In: Rhodes G, Calder A, Johnson M, Haxby JV, editors. Oxford Handbook of Face Perception. Oxford University Press.

Fusar-Poli P, Placentino A, Carletti F, Landi P, Allen P, Surguladze S, Benedetti F, Abbamonte M, Gasparotti R, Barale F, Perez J, McGuire P, Politi P. 2009. Functional atlas of emotional faces processing: a voxel-based meta-analysis of 105 functional magnetic resonance imaging studies. J Psychiatry Neurosci 34:418-432.

George N, Dolan RJ, Fink GR, Baylis GC, Russell C, Driver J. 1999. Contrast polarity and face recognition in the human fusiform gyrus. Nat Neurosci. 2:574-580.

Graham R, Labar KS. 2012. Neurocognitive mechanisms of gaze-expression interactions in face processing and social attention. Neuropsychologia 50:553-566.

Gramfort A, Papadopoulo T, Olivi E, Clerc M. 2010. OpenMEEG: opensource software for quasistatic bioelectromagnetics. Biomed Eng Online 9:45.

Gur RC, Schroeder L, Turner T, McGrath C, Chan RM, Turetsky BI, Alsop D, Maldjian J, Gur RE. 2002. Brain activation during facial emotion processing. Neurolmage 16:651-662.

Habel U, Windischberger C, Derntl B, Robinson S, Kryspin-Exner I, Gur RC, Moser E. 2007. Amygdala activation and facial expressions: explicit emotion discrimination versus implicit emotion processing. Neuropsychologia 45:2369-2377. 
Hadj-Bouziane F, Liu N, Bell AH, Gothard KM, Luh W-M, Tootell RBH, Murray EA, Ungerleider LG. 2012. Amygdala lesions disrupt modulation of functional MRI activity evoked by facial expression in the monkey inferior temporal cortex. Proc Natl Acad Sci 109:E3640-3648.

Hadjikhani N, Hoge R, Snyder J, de Gelder B. 2008. Pointing with the eyes: the role of gaze in communicating danger. Brain Cogn 68:1-8.

Haxby, Hoffman, Gobbini. 2000. The distributed human neural system for face perception. Trends Cogn Sci. 4:223-233.

Hein G, Knight RT. 2008. Superior temporal sulcus--It's my area: or is it? J Cogn Neurosci 20:21252136.

Henson RN, Goshen-Gottstein Y, Ganel T, Otten LJ, Quayle A, Rugg MD. 2003. Electrophysiological and haemodynamic correlates of face perception, recognition and priming. Cereb Cortex 13:793805.

Hoffman EA, Haxby JV. 2000. Distinct representations of eye gaze and identity in the distributed human neural system for face perception. Nat Neurosci 3:80-84.

Höistad M, Barbas H. 2008. Sequence of information processing for emotions through pathways linking temporal and insular cortices with the amygdala. Neurolmage 40:1016-1033.

Itier RJ, Latinus M, Taylor MJ. 2006. Face, eye and object early processing: what is the face specificity? Neurolmage. 29:667-676.

Itier RJ, Taylor MJ. 2002. Inversion and contrast polarity reversal affect both encoding and recognition processes of unfamiliar faces: a repetition study using ERPs. Neurolmage. 15:353-372. Itier RJ, Taylor MJ. 2004. Source analysis of the N170 to faces and objects. Neuroreport. 15:12611265. 
Kanwisher N, McDermott J, Chun MM. 1997. The fusiform face area: a module in human extrastriate cortex specialized for face perception. J Neurosci Off J Soc Neurosci. 17:4302-4311.

Keightley ML, Winocur G, Graham SJ, Mayberg HS, Hevenor SJ, Grady CL. 2003. An fMRI study investigating cognitive modulation of brain regions associated with emotional processing of visual stimuli. Neuropsychologia 41:585-596.

Klucharev V, Sams M. 2004. Interaction of gaze direction and facial expressions processing: ERP study. Neuroreport 15:621-625.

Kybic J, Clerc M, Abboud T, Faugeras O, Keriven R, Papadopoulo T. 2005. A common formalism for the integral formulations of the forward EEG problem. IEEE Trans Med Imaging 24:12-28.

Lahnakoski JM, Glerean E, Salmi J, Jääskeläinen IP, Sams M, Hari R, Nummenmaa L. 2012. Naturalistic FMRI mapping reveals superior temporal sulcus as the hub for the distributed brain network for social perception. Front Hum Neurosci 6:233.

Liu Y, Huang H, McGinnis-Deweese M, Keil A, Ding M. 2012. Neural substrate of the late positive potential in emotional processing. J Neurosci 32:14563-14572.

Machens CK, Gollisch T, Kolesnikova O, Herz AVM. 2005. Testing the efficiency of sensory coding with optimal stimulus ensembles. Neuron 47:447-456.

N’Diaye K, Sander D, Vuilleumier P. 2009. Self-relevance processing in the human amygdala: gaze direction, facial expression, and emotion intensity. Emotion 9:798-806.

Phillips ML, Drevets WC, Rauch SL, Lane R. 2003. Neurobiology of emotion perception I: The neural basis of normal emotion perception. Biol Psychiatry 54:504-514.

Pourtois G, Sander D, Andres M, Grandjean D, Reveret L, Olivier E, Vuilleumier P. 2004. Dissociable roles of the human somatosensory and superior temporal cortices for processing social face signals. Eur J Neurosci 20:3507-3515. 
Puce A, Allison T, Bentin S, Gore JC, McCarthy G. 1998. Temporal cortex activation in humans viewing eye and mouth movements. J Neurosci 18:2188-2199.

Rigato S, Farroni T, Johnson MH. 2010. The shared signal hypothesis and neural responses to expressions and gaze in infants and adults. Soc Cogn Affect Neurosci 5:88-97.

Rossion B, Joyce CA, Cottrell GW, Tarr MJ. 2003. Early lateralization and orientation tuning for face, word, and object processing in the visual cortex. Neurolmage 20:1609-1624.

Rushworth MFS, Passingham RE, Nobre AC. 2002. Components of switching intentional set. J Cogn Neurosci 14:1139-1150.

Sander D, Grandjean D, Kaiser S, Wehrle T, Scherer KR. 2007. Interaction effects of perceived gaze direction and dynamic facial expression: Evidence for appraisal theories of emotion. Eur J Cogn Psychol 19:470-480.

Sato W, Kochiyama T, Uono S, Yoshikawa S. 2010. Amygdala integrates emotional expression and gaze direction in response to dynamic facial expressions. Neurolmage 50:1658-1665.

Sato W, Yoshikawa S, Kochiyama T, Matsumura M. 2004. The amygdala processes the emotional significance of facial expressions: an fMRI investigation using the interaction between expression and face direction. Neurolmage 22:1006-1013.

Schilbach L, 2010. A second-person approach to other minds. Nat Rev Neurosci 11: 449.

Schupp HT, Cuthbert BN, Bradley MM, Cacioppo JT, Ito T, Lang PJ. 2000. Affective picture processing: the late positive potential is modulated by motivational relevance. Psychophysiology 37:257-261.

Tadel F, Baillet S, Mosher JC, Pantazis D, Leahy RM. 2011. Brainstorm: a user-friendly application for MEG/EEG analysis. Comput Intell Neurosci 2011:879716. 
Tzourio-Mazoyer N, Landeau B, Papathanassiou D, Crivello F, Etard O, Delcroix N, Mazoyer B, Joliot M. 2002. Automated anatomical labeling of activations in SPM using a macroscopic anatomical parcellation of the MNI MRI single-subject brain. Neurolmage 15:273-289.

Vlamings PHJM, Goffaux V, Kemner C. 2009. Is the early modulation of brain activity by fearful facial expressions primarily mediated by coarse low spatial frequency information? J Vis 9:12.1-13.

Watanabe S, Kakigi R, Puce A. 2003. The spatiotemporal dynamics of the face inversion effect: A magneto- and electro-encephalographic study. Neuroscience. 116:879-895.

Zaki J, Weber J, Ochsner K. 2012. Task-dependent neural bases of perceiving emotionally expressive targets. Front Hum Neurosci. 6:228. 


\section{FIGURE LEGENDS}

Figure 1. Experimental procedure and stimuli examples. a) From the initial position, gaze, emotion and gesture were manipulated: stimuli displayed a direct or an averted gaze, an angry or a neutral expression, a pointing gesture or not. b) Trial procedure: a central fixation area where the face of the stimuli later appears was presented for $500 \mathrm{~ms}$. In the Expression task blocs, subjects had to judge whether the actor displayed an angry or a neutral expression. In the Direction task blocs, they had to judge whether the actor was addressing them or another.

Figure 2. Social cues interaction on the N170 as a function of self-relevance. a) Post-test selfinvolvement ratings on a scale from 0 to 9. Description of the conditions from left to right: 1 . Neutral, direct gaze, no pointing; 2 . Neutral, direct gaze, pointing; 3 . Angry, direct gaze, no pointing; 4 . Angry, direct gaze, pointing; 5. Neutral, averted gaze, no pointing; 6. Neutral, averted gaze, pointing; 7. Angry, averted gaze, no pointing; 8 . Angry, averted gaze, pointing. b) Amplitude of the N170 activity averaged between 162 and 186 ms in microVolts. Same order of conditions as above. c) N170 interaction between emotion, gaze and gesture. Neural activity and self-involvement ratings increased with the number of self-oriented social signals. ${ }^{*} p<0.05$; ${ }^{* * *} p<0.001$; ns Nonsignificant.

Figure 3. Neural sources of the $\mathbf{N} 170$ interaction. Regions that reflect the parametric increase of selfrelevance with the number of self-oriented social signals. Both tasks (grey) involved temporal and occipital regions, including the fusiform gyrus, whereas the Expression task (green) essentially involved regions in the limbic system, including the amygdala, and the Direction task (blue) regions in the dorsal pathway, including the parietal. For the three-mentioned example, the activity of a representative vertex is plotted (pA.m) for the Direction task (blue) and for the Expression task (green) separately for comparison. The order of the conditions for each plot is similar to the one of Figure 2. ${ }^{*} p<0.05$ 
Supplementary figure S1. Main effects observed on P100, P200 and LPP. a) P100 topography at the time around the peak amplitude (92-116 ms) and main effects plots of emotion and gesture on electrode PO8. b) P200 topography at the time around the peak amplitude(184-208 ms) and main effects plots of emotion and gaze on electrode Cz. c) LPP topography between 300 and 700 ms and main effects plots of emotion and task on electrode Pz. ${ }^{*} p<0.05 ;{ }^{* *} p<0.01 ;{ }^{* * *} p<0.001$.

\section{TABLE}

Table 1. Number of vertices $\left(>15\right.$, surface area $\left.>1.5 \mathrm{~cm}^{2}\right)$ for each region reported in source localization analysis based on the correspondence between the Tzourio-Mazoyer brain atlas, the brainstorm generic brain and source model implemented in Brainstorm software. $R / L$ : $R=$ Right hemisphere, L= Left Hemisphere

$E / D$ : $E=$ Regions involved mainly in the Expression task, $D=$ Regions involved mainly in the Direction task. 
Table 1. Number of vertices for each region reported as integrating emotion, gaze and gesture.

\begin{tabular}{|c|c|c|c|}
\hline Task & Hemisphere & Brain regions & Vertices \\
\hline$E / D$ & $\mathrm{R}$ & Fusiform & 21 \\
\hline$E / D$ & $\mathrm{R}$ & Middle occipital & 36 \\
\hline$E / D$ & $\mathrm{R}$ & Middle temporal & 103 \\
\hline$E / D$ & $\mathrm{R}$ & Inferior occipital & 36 \\
\hline $\mathrm{D}$ & $\mathrm{L}$ & Postcentral & 18 \\
\hline D & $\mathrm{L}$ & Inferior/superior parietal & 36 \\
\hline$E$ & $R / L$ & Subgenual cingulate & $34 / 23$ \\
\hline$E$ & $R / L$ & Rectus & $16 / 35$ \\
\hline$E$ & $R / L$ & Hippocampus & $41 / 38$ \\
\hline$E$ & $R / L$ & Amygdala & $23 / 26$ \\
\hline$E$ & $R / L$ & Caudate & $80 / 64$ \\
\hline$E$ & L & Inferior frontal & 23 \\
\hline$E$ & $\mathrm{~L}$ & Thalamus & 16 \\
\hline$E$ & $\mathrm{~L}$ & Superior Temporal Pole & 16 \\
\hline $\mathrm{E}$ & $\mathrm{L}$ & Calcarine fissure & 16 \\
\hline
\end{tabular}




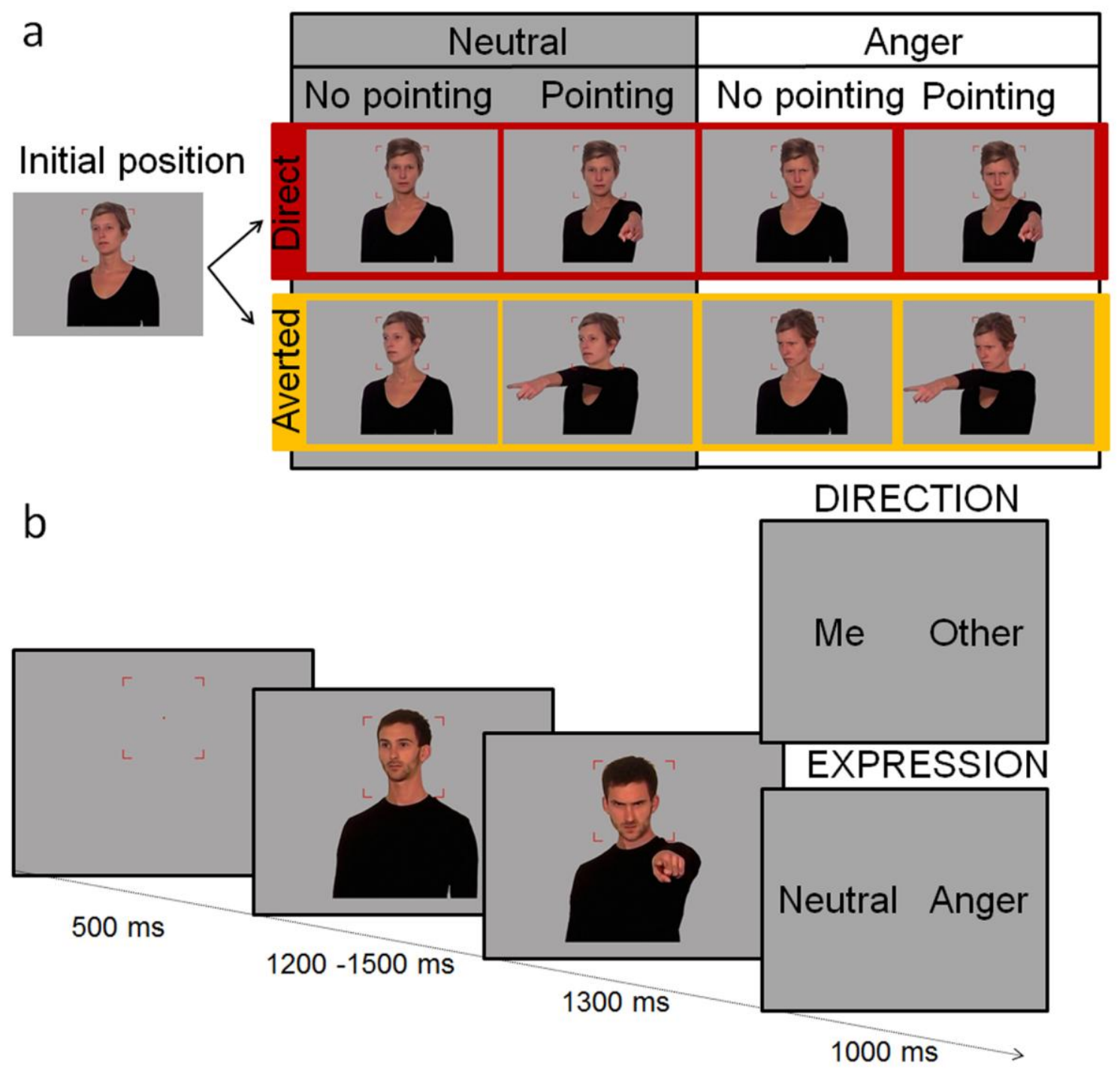

Figure 1 

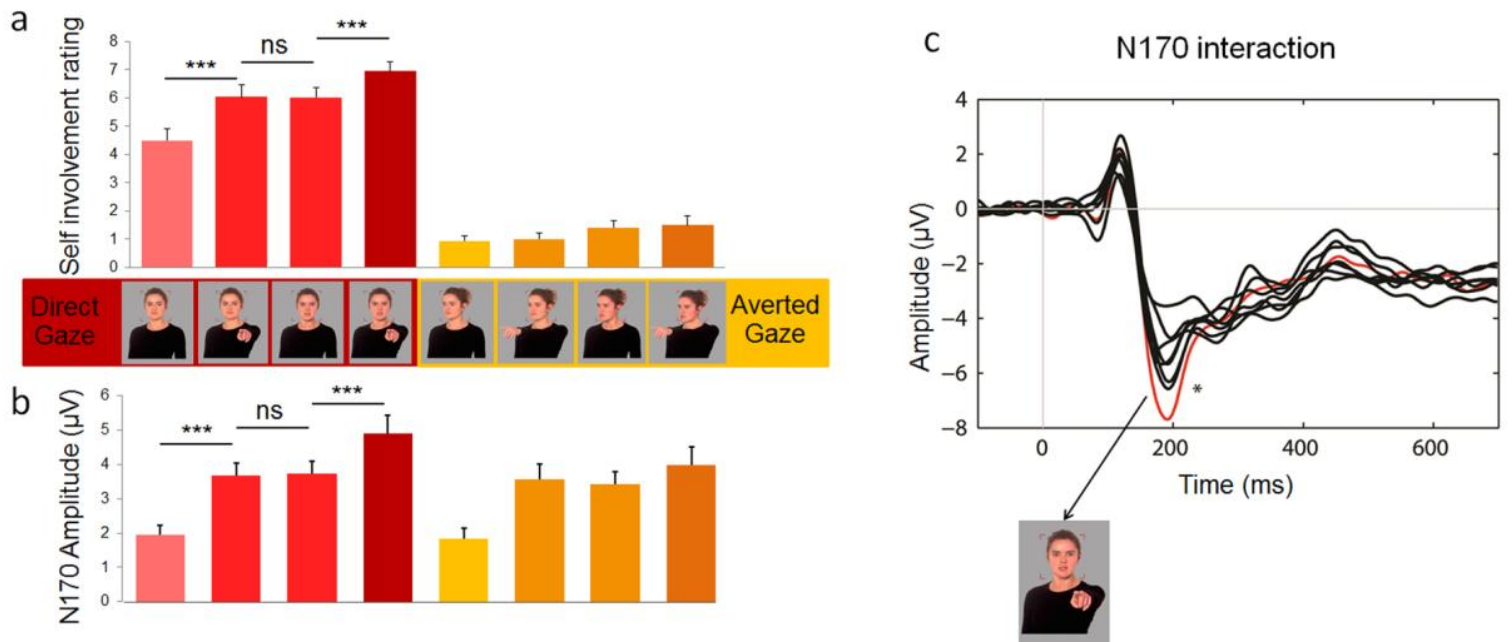

Figure 2 


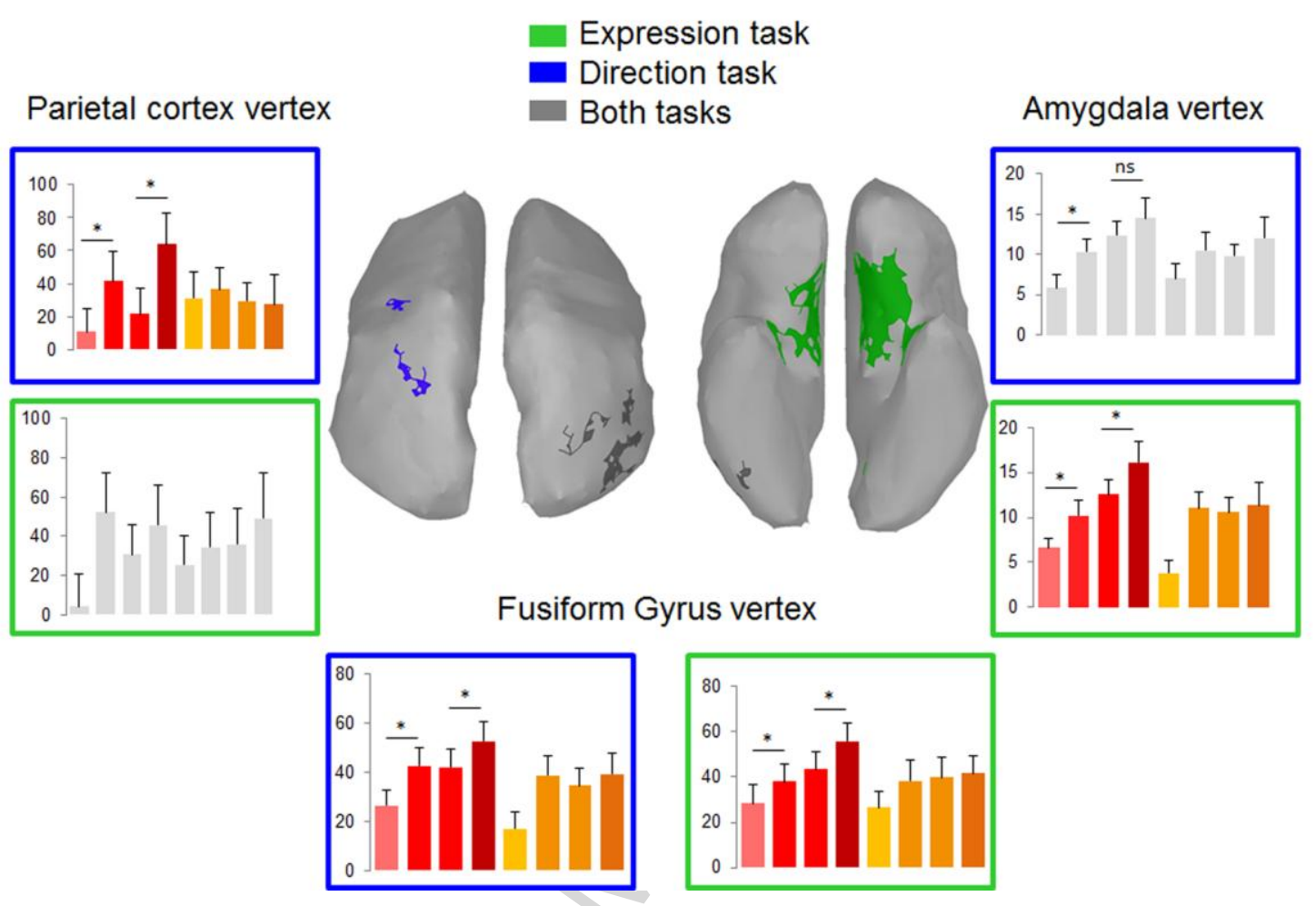

Figure 3 
Highlights

- A temporal marker of social cues integration emerges $170 \mathrm{~ms}$ after the stimulus onset.

- This temporal marker appears to be independent of selective attention.

- The brain sources are partly modulated as a function of attended social cue.

- Co-emitted social cues are integrated as long as they are relevant to the observer. 